
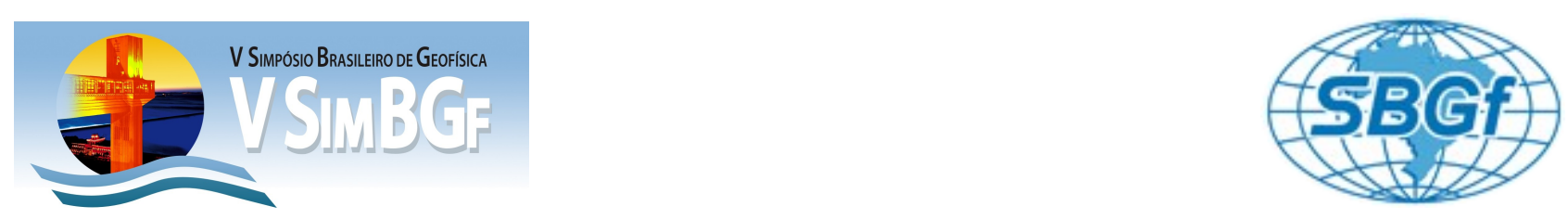

\title{
Sistema de Informação Geográfico para Análise de dados Sismológicos
}

Helmuth Saatkamp, Departamento de Ciência da Computação, UnB

Henrique Pereira de Freitas Filho, Departamento de Ciência da Computação, UnB

Maristela Holanda, Departamento de Ciência da Computação, UnB

George Sand França, Observatório Sismológico, Instituto de Geociências, UnB

Copyright 2012, SBGf - Sociedade Brasileira de Geofísica

Este texto foi preparado para a apresentação no $V$ Simpósio Brasileiro de Geofísica, Salvador, 27 a 29 de novembro de 2012. Seu conteúdo foi revisado pelo Geolisica, Salvador, 27 a 29 de novento de 2012. Seu conteúdo for revisado pelo SBGf ou de seus associados. É proibida a reproducão total ou parcial deste aprodução total ou parc material para propósitos comerciais sem prévia autorização da SBGf.

\section{Abstract}

An important question related to seismic data is the need to have access to them quickly and easily. In this context, this job presents a Geographic Information System on the web, comprising the geographic database servers and interactive maps, with the aim of assisting in the recovery and presentation of seismological data. The system was designed and implemented for data analysis in the Seismological Observatory of the University of Brasilia.

\section{Introdução}

Os terremotos fornecem diversas informações que são necessárias para que orgãos de defesa, meios de comunicações e pesquisadores possam ter acesso de forma prática e rápida. Aliada a isso também, os analistas precisam uma maior quantidade de informação para os relatórios técnicos e informativos do próprio Observatório. Assim, iniciou-se o estudo de implementação de um banco de dados para atender ao Observatório Sismológico verificando sua particularidade e integrando no "padrão internacional" de dados sismológicos.

Os dados do sismograma deve ser armazenados em arquivos no formato miniSEED (Standard for the Exchange of Earthquake Data). O formato miniSEED é composto por um cabeçalho que tem um conjunto de metadados que são utilizados para facilitar a recuperação dos dados sismológicos

Para a organização dos dados foi utilizado um Sistema Gerenciador de Banco de Dados (SGBD) que é um software que facilita a construção e manutenção de diferentes bancos de dados. Foi estabelecido um modelo de banco de dados para os dados sismológicos que foi implementado em um SGBD, com o objetivo de facilitar o acesso e a correlação entre os dados.

Em relação à facilidade de acesso e uso dos dados coletados, foi desenvolvido um sistema de informação geográfico na web, SIG/Web, com servidores de mapas interativos, no qual, de maneira simples o usuário da informação pode ter acesso aos dados desejados.
Esse trabalha tem como objetivo apresentar um banco de dados que modela a análise dos fenômenos sismológicos, assim como também um Sistema de Informação Geográfico na web para o fácil acesso dos dados.

\section{Sistema de Informação Geográfico na Web}

Um sistema é um conjunto de elementos ou componentes independentes que interagem para atingir um objetivo, ou seja, o que caracteriza um sistema são os seus componentes e as relações entre eles para alcançar uma meta comum (Bertalanffy, 1975). Já um sistema de informação é um sistema cujo objetivo é a coleta, a manipulação, o armazenamento e a disseminação de dados e informações (Côrtes, 2008) (Kobiyam et al., 2006) (Laudon \& Laudon, 1999). Um Sistema de Informação Geográfico (SIG) é um sistema de informação que realiza o tratamento computacional de dados geográficos.

SIG Web é qualquer SIG que usa tecnologias da Web. A forma mais simples de SIG Web deve ter pelo menos um servidor e um cliente, onde o servidor é um servidor de aplicações Web e o cliente é um navegador Web ou uma aplicação desktop ou uma aplicação de celular (Bressan, 2010) (Fu \& Sun, 2010).

No que diz respeito à arquitetura de um SIG Web, a mais comumente usada é baseada em três camadas: Camada de Interface, Camada de Servidor de Aplicação e a Camada de Banco de Dados (Baumann, 2011) (Shunfu, 2002) (Frehner M. \& Brandli, 2006).

A Camada de Interface serve como uma interface de usuário gráfica para apresentar o resultado dos dados espaciais, permitindo aos utilizadores finais interagir com os serviços oferecidos pelo SIG Web.

A Camada de Servidor de Aplicação se comunica com múltiplas fontes de dados e interage com os usuários finais para analisar e manipular dados provenientes de serviços de provedor de dados.

A Camada de Banco de Dados é um conjunto de serviços provedor de dados remotos que são utilizados pelo SIG Web. Cada banco de dados prestador de serviço oferece um conjunto de interfaces através das quais aplicações clientes podem utilizar e manipular os dados remotamente. Essa camada utiliza-se de banco de dados geográficos que é uma coleção de dados relacionados entre si que tem atributos convencionais e atributos geográficos. 


\section{SIGweb do Observatório Sismológico da Universidade de Brasília}

A arquitetura abstrata proposta para o SIGweb do Observatório Sismológico da Universidade de Brasília é ilustrada na Figura 1, sendo composta por três grandes módulos: Camada de Interface, que é responsável pela interação do sistema com o usuário através de Formulários e Mapas; a Camada de Aplicação, responsável pelo mecanismo de geração dos mapas; e por fim, a Camada de Persistência, onde os dados são tratados e armazenados em um banco de dados.

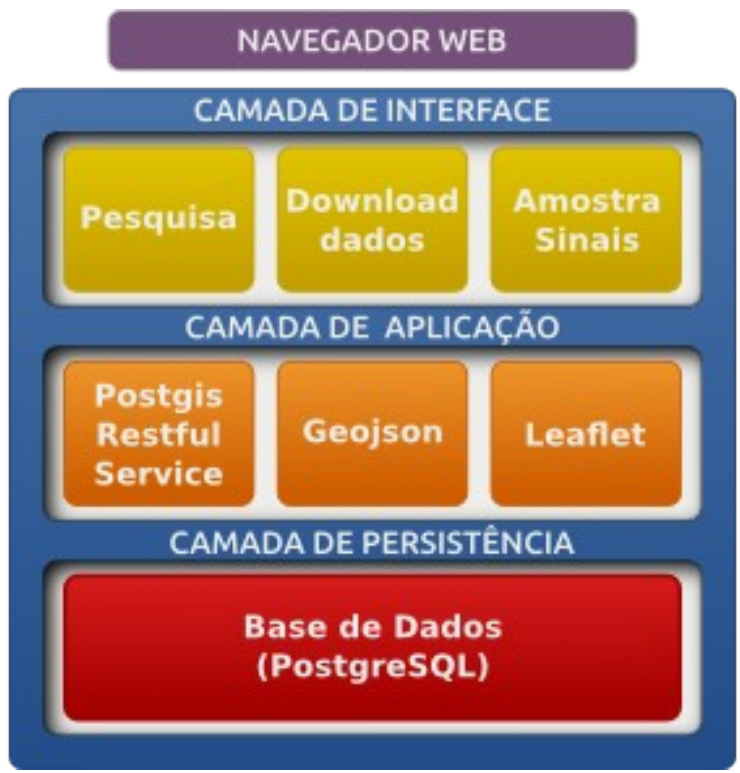

Figura 1 - Arquitetura Abstrata do SIGweb

A camada de interface é composta pelos módulos: Pesquisa, onde o usuário pode pesquisar por cidade, magnitude e intervalo de datas; Download de dados, onde um arquivo miniSEED é inserido no banco de dados; Amostra de sinais uma imagem poderá ser visualizada referente ao evento sísmico.

A camada de Aplicação é composta por: O PostGIS RESTful Web Service é um Framework em PHP criado para prover uma série de serviços web em análise espacial dos dados em Bancos PostreSQL+PostGIS. A saída é especificado pelo usuário como parte da chamada REST e pode ser XML, JSON, ou JSONP; GeoJSON é um formato aberto para codificar uma variedade de estruturas de dados geográficos. Baseado em JSON (JavaScript Object Notation). O GeoJSON permite que os dados geográficos sejam armazenados de forma legível que é geralmente mais compacto que o XML. Tipos de dados espaciais suportados pelo GeoJSON incluem pontos, polígonos, multipolígonos, características, coleções de geometrias e caixas delimitadoras, que são armazenados junto com as informações e os atributos. O Leaflet é uma biblioteca opensource em JavaScript para criação de mapas interativos aproveitando as novas tecnologias em navegadores modernos. O Leaftlet faz uso do GeoJSON.

A camada de persistência é responsável pelos dados do sistema, sendo dividida em duas partes principais: o SGBD Postgre com extensão espacial PostGIS que armazena os dados do sistema.

\section{Funcionamento do SIGweb}

A Figura 2 ilustra o funcionamento do Leaflet. Ao ser solicitado por um usuário, o Leaflet aciona a API e carrega os mapa, após essa etapa é feito uma requisição ao servidor pelas definições de georeferência, o servidor acessa o banco de dados e retorna a consulta onde é utilizada pelo leaflet para exibição dos dados requisitados, a renderização se faz pela api do Leaflet utilizando tecnologias como HTML5 e CSS3.

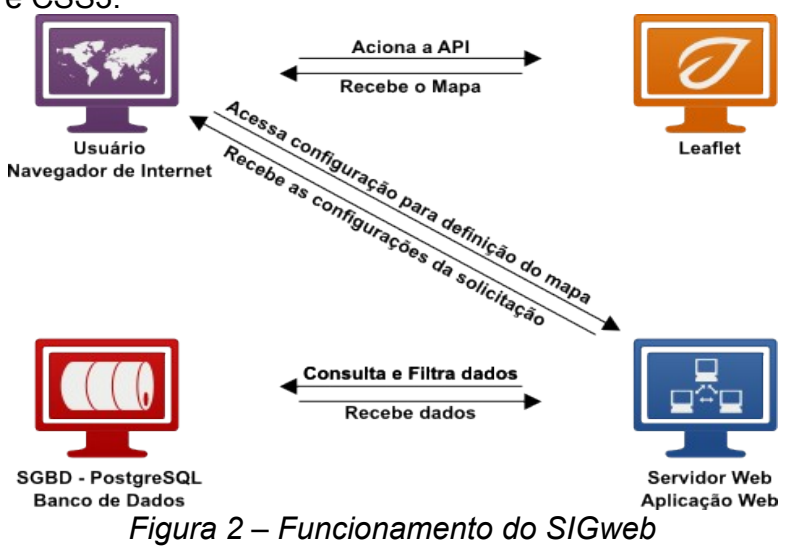

\section{Modelo de Dados do Banco de Dados}

Para a criação do modelo conceitual foi utilizado o modelo de dados OMT-G. Esse modelo foi escolhido seguindo a especificação da INDE (Infraestrutura Nacional de Dados Espaciais) que é o conjunto integrado de tecnologias, políticas, mecanismos e procedimentos de coordenação e monitoramento; padrões e acordos, necessário para facilitar e ordenar a geração, o armazenamento, o acesso, o compartilhamento, a disseminação e o uso dos dados geoespaciais de origem federal, estadual, distrital e municipal no Brasil (Comitê de Planejamento da INDE, 2010) (Borges et al., 2001).

A partir dos estudos sobre os metadados dos arquivos dos dados sismológico foi definido inicialmente um modelo composto por 11 entidades: proprietário, rede, estação, estado, província tectônica, canal, dado bruto, dado analisado, analista, evento e tipo de evento.

Na Figura 3 o modelo conceitual baseado no OMT-G é apresentado. O OMT-G é o padrão definido pela INDE para modelagem de dados conceituais. 


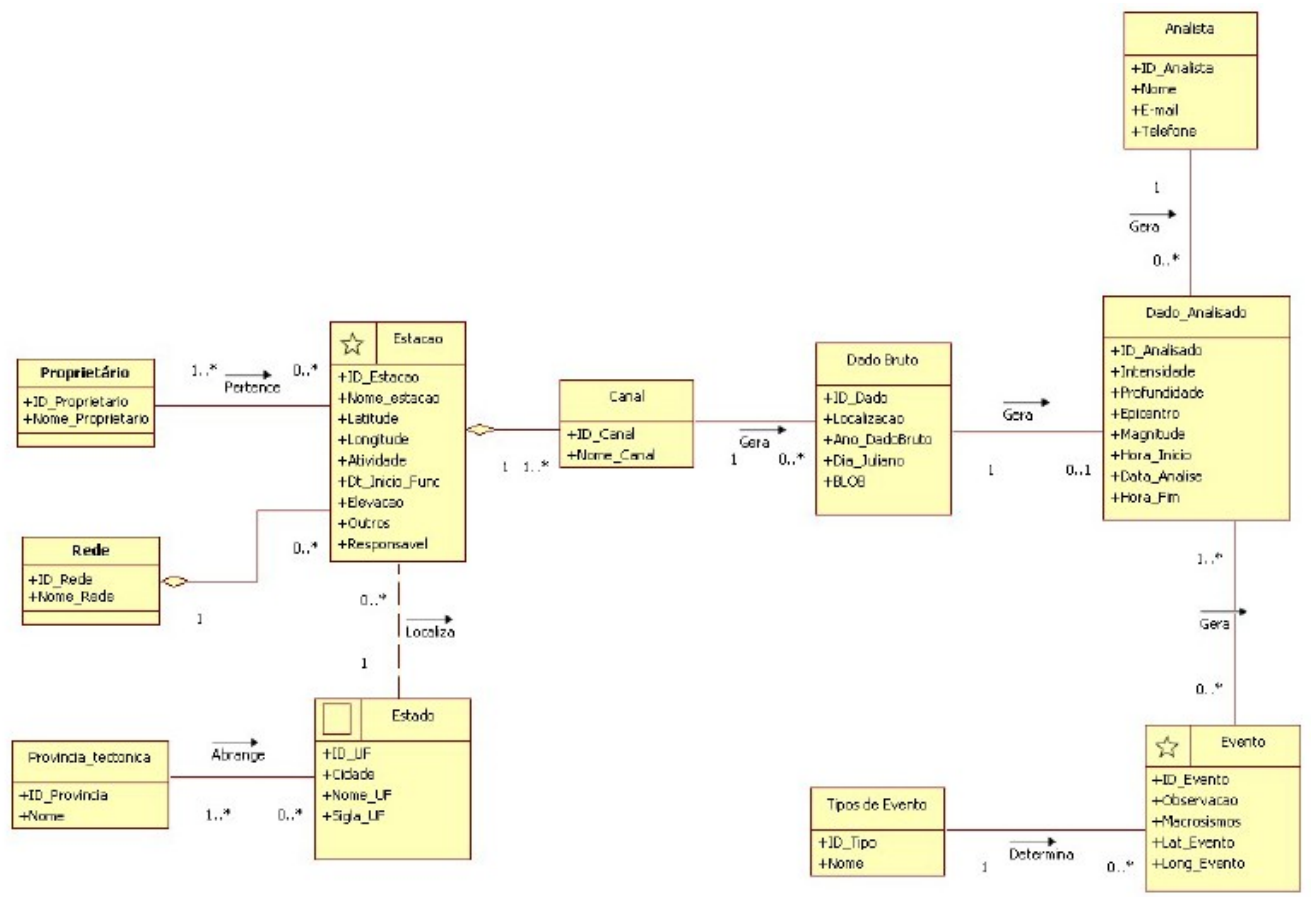

Figura 3 - Modelo de Dados OMT-G dos Dados de Fenômenos Sismol

\section{Tecnologias Utilizadas}

O SIG web segue a arquitetura cliente/servidor, ou seja, computadores clientes requisitam serviços de computadores servidores que provê serviços. Os dados estão armazenados em um banco de dados geográfico que foi implementado no SGBD PostGis, que é o SGBD PostgreSQL com uma extensão espacial.

Para o desenvolvimento da interface do sistema foi utilizada a linguagem de programação PHP. Para as validações do sistema foi utilizada a linguagem de programação Javascript. Para a visualização dos dados em mapas foi utilizado o Leaflet.

A Figura 4 apresenta a página inicial do sistema onde as estações são representadas por triângulos verdes $\mathrm{e}$ os dados analisados são os pontos vermelhos. Esses elementos estão georeferenciados no mapa do Brasil. Essa ferramenta permite expandir o mapa até o nível de ruas. Se o usuário desejar é possível apresentar apenas as estações, assim como também apenas os pontos analisados.

A Figura 5 apresenta os dados sísmicos referentes a um evento ocorrido no Pará, juntamente com o nome de uma estação que captou o fenômeno sísmico.

A Figura 6 ilustra o resultado da busca ao ser selecionado apenas eventos que tenha magnitude maior que 4.

A Figura 7 ilustra o resultado da busca ao ser selecionada eventos no estado de Minas Gerais. 


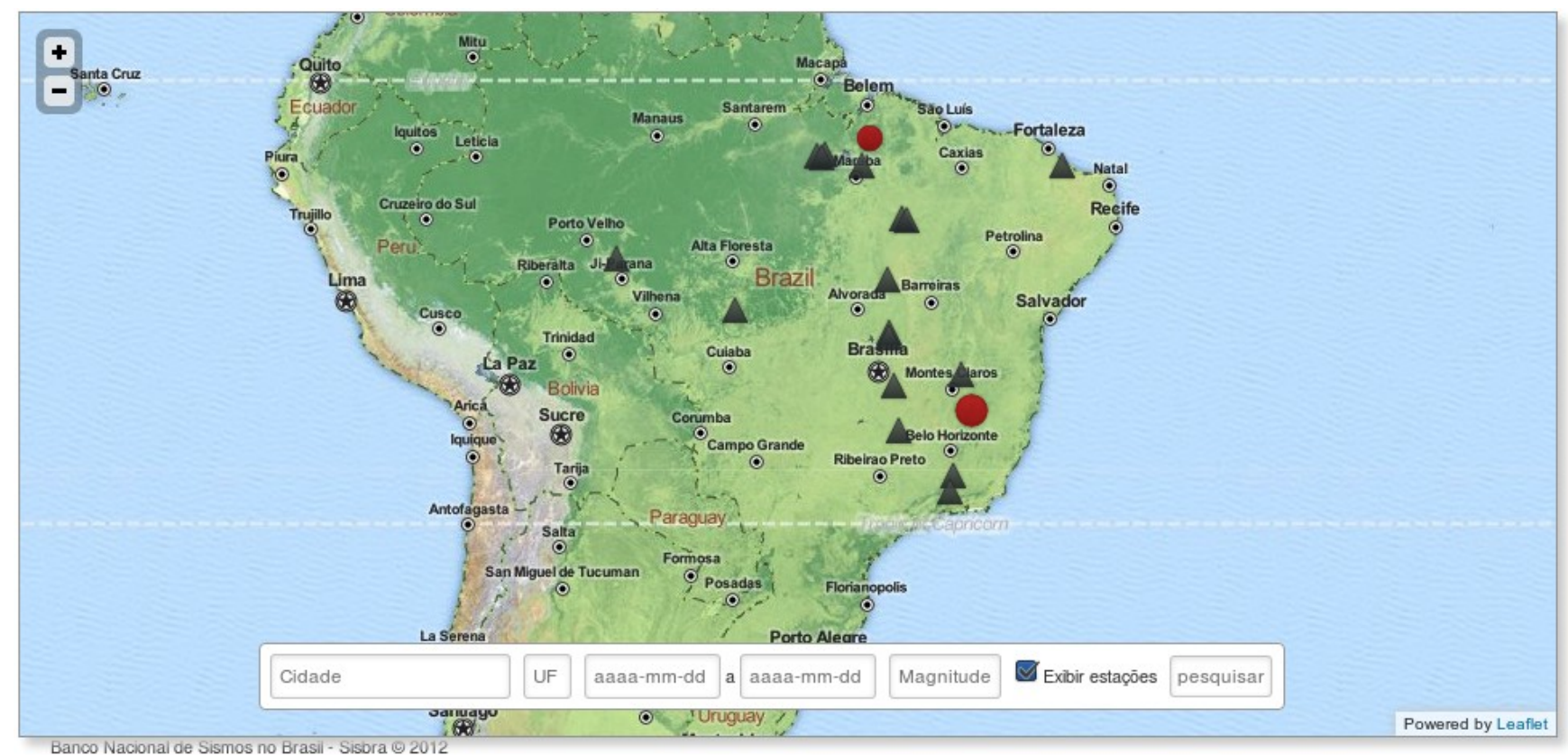

Figura 4 - Página Inicial do SIGweb

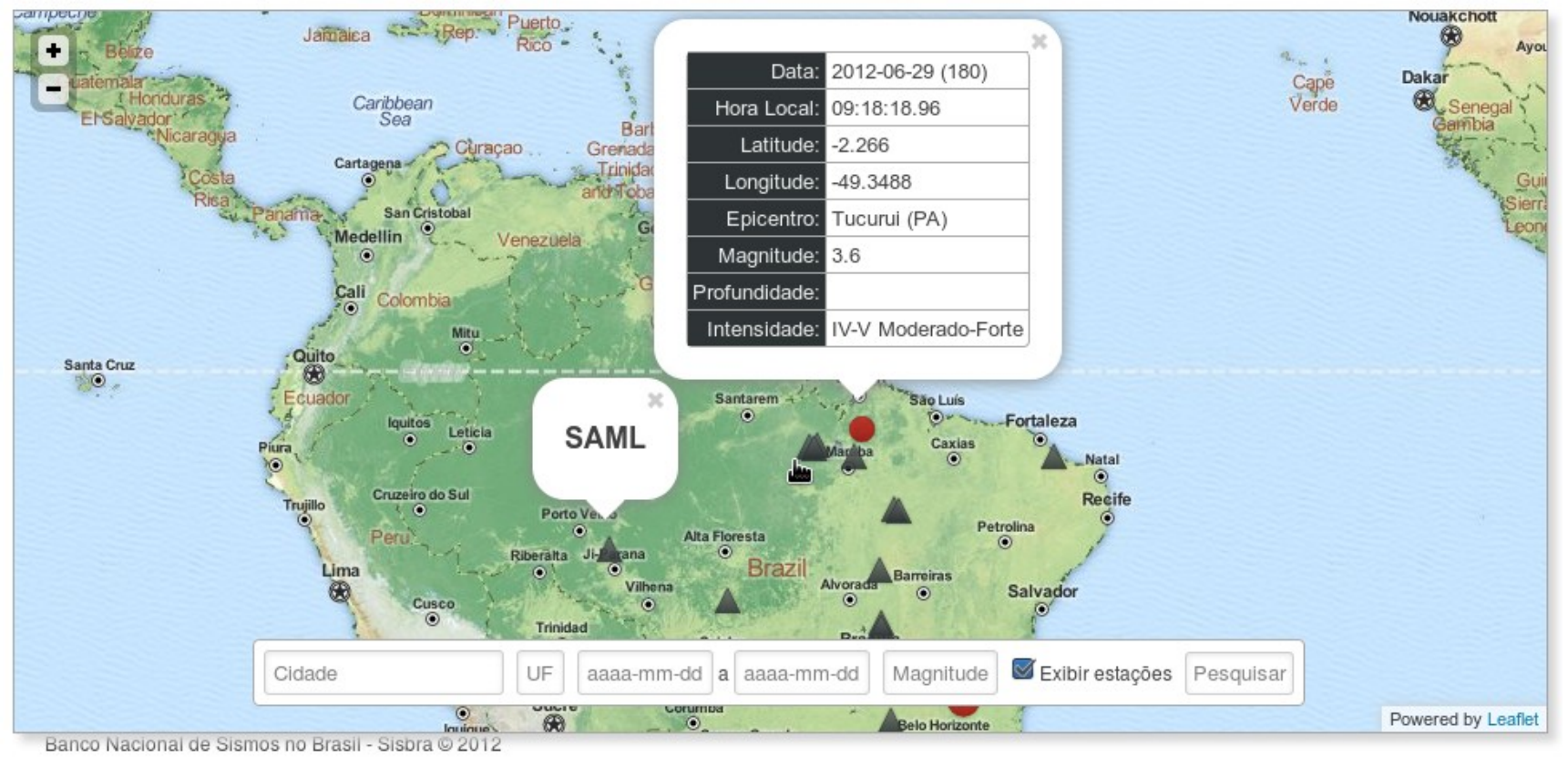

Figura 5 - Imagem de uma Estação Especifica e um evento sísmico. 


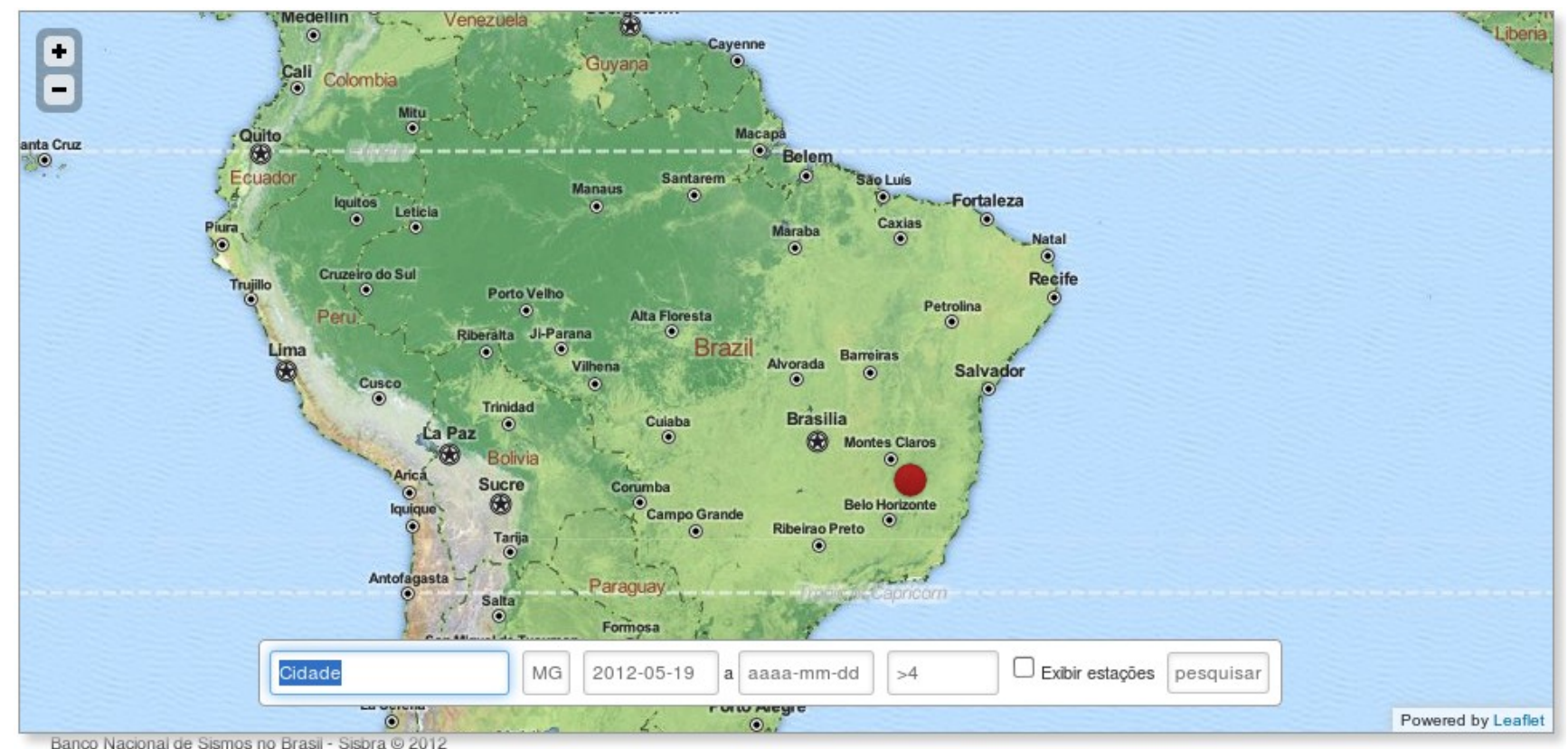

Figura 6 - Eventos com magnitude maior que 4.

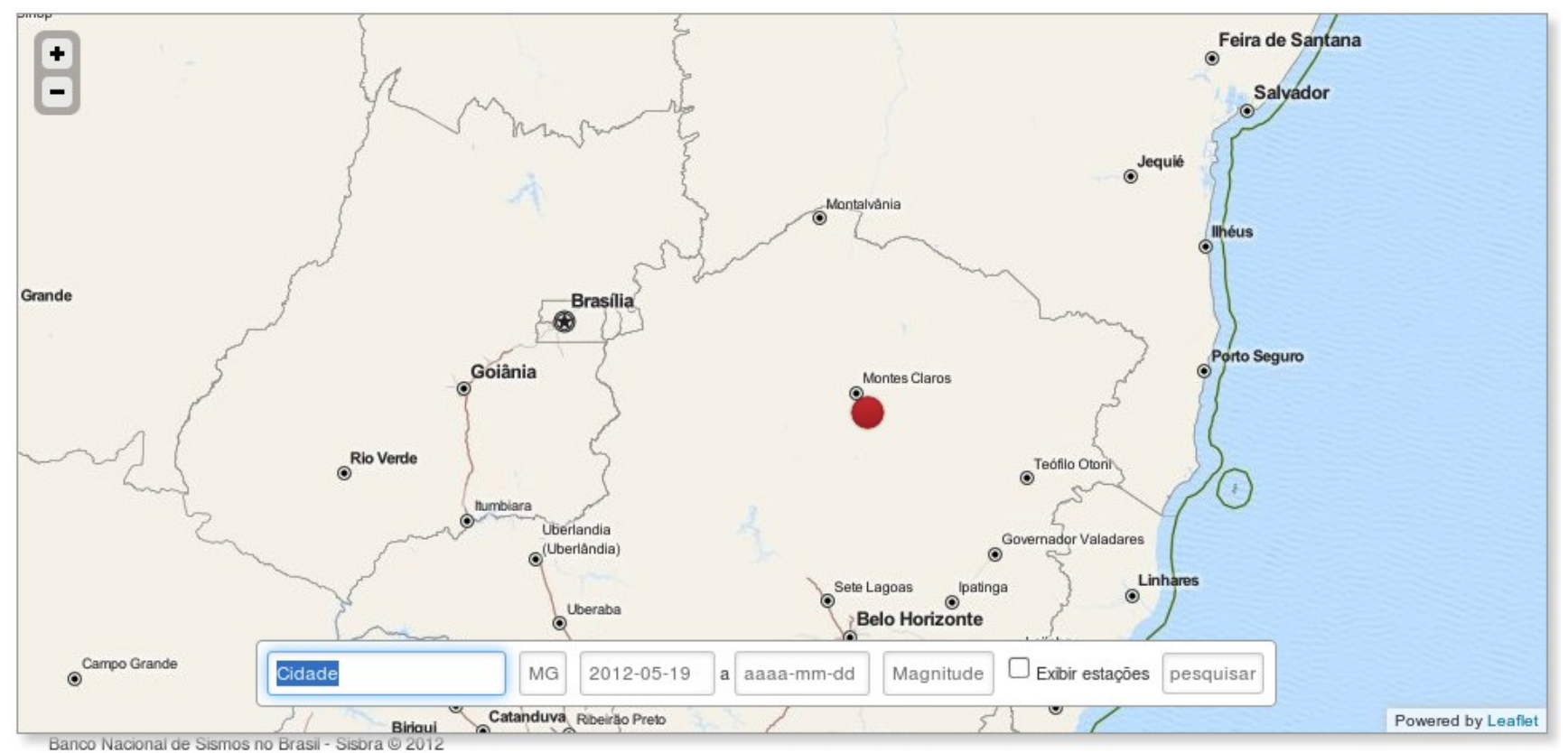

Figura 7 - Evento no estado de Minas Gerais.

\section{Discussão e Conclusões}

Atualmente o Observatório Sismológico recebe um volume enorme de dados gerados a partir dos sismógrafos, que são armazenados em máquinas sem está organizado em um sistema de informação que facilite a busca, a recuperação e a análise desses dados e sem está georeferenciado que facilita a análise dos dados. Para a solução desse problema foi desenvolvido um SIG Web com o objetivo de melhorar o processo de análise e a organização dos dados sismológicos. O SIG web do Observatório Sismológico de Brasília é composto por um banco de dados geográfico para organizar o armazenamento dos dados sismológicos e facilitar uma futura busca desses dados, a funcionalidade para disponibilização do arquivo miniSEED para o analista de forma automática e a apresentação dos dados sismológicos com a visualização dos eventos gerados no mapa.

Os analistas do Observatório Sismológico que fazem um processo manual de análise dos dados sismológicos, podem fazer esse processo de forma rápida, eficiente e com uma certa automatização, melhorando o processo de trabalho do analista, o armazenamento dos dados sismológicos e a qualidade da análise dos dados .

\section{Agradecimentos}

Os autores agradecem ao apoio do $\mathrm{CIC} / \mathrm{UnB}$, ao Observatório Sismológico/UnB e aos técnicos responsáveis pela análise de dados. GSF agradece a CNPq pelo apoio, através da bolsa PQ (processo 303529/2010-5) 


\section{Referências}

Bertalanffy L. V., 1975. Teoria Geral dos Sistemas. Editora Vozes.

Borges K. A. V., Davis JR C. A. \& Laender A. H. F., 2001. Omt-g: An object-oriented data model for geographic applications. Geolnformatica. Kluwer Academic Publishers.

Bressan T., 2010. Desenvolvimento e integração de um ambiente SIGWEB com ferramentas de software livre. Dissertação de mestrado. Universidade Federal de Santa Maria, Brasil.

Comitê de Planejamento da Infraestrutura Nacional de Dados Espaciais INDE, 2010. Plano de Ação para Implantação da INDE - Infraestrutura Nacional de Dados Espaciais. Ministério do Planejamento, Orçamento e Gestão, Rio de Janeiro, Brasil.

Côrtes P. L., 2008. Administração de Sistemas de Informação. Editora Saraiva.

Fu P. \& Sun J., 2010. Web GIS: Principles and Applications. ESRI Press.
Joomla, 2006. What is Joomla?. Disponível em: <http://www.joomla.org/about-joomla.html>. Acesso em: 10 dez. 2011.

Kobiyam M., Mendonça M., Moreno D. A., Marcelino I. P. V. O., Marcelino A. V., Gonçalves E. F., Brazetti L. L. P., Oerl R. F., Molleri G. S. F. \& Rudorff F. M., 2006. Prevenção de Desastres Naturais - Conceitos Básicos. Organic Trading, Santa Catarina, Brasil.

Laudon K. C. \& Laudon J. P., 1999. Sistemas de Informação com Internet. LTC, Rio de Janeiro, Brasil.

Baumann J., 2011. "Future of Web GIS: An Interview with Pinde Fu". GeoConnection International Magazine.

Shunfu H., 2002. "Web-Based Multimedia GIS for the analysis and visualization of spatial environmental database". Proc. Symposium on Geospatial Theory, Processing and Applications.

Frehner M. \& Brandli M., 2006. "Virtual database: Spatial analysis in a Web-based data management system for distributed ecological data". Environmental Modelling \& Software, vol. 21, pp. 1544-1554, doi:10.1016/j.envsoft.2006.05.012. 